This item was submitted to Loughborough's Research Repository by the author.

Items in Figshare are protected by copyright, with all rights reserved, unless otherwise indicated.

\title{
Diffraction of water waves by a segmented permeable breakwater
}

PLEASE CITE THE PUBLISHED VERSION

LICENCE

CC BY-NC-ND 4.0

\section{REPOSITORY RECORD}

Mclver, P.. 2019. "Diffraction of Water Waves by a Segmented Permeable Breakwater". figshare. https://hdl.handle.net/2134/247. 


\title{
Diffraction of water waves by a segmented permeable breakwater
}

\author{
P. McIver*
}

\begin{abstract}
The linearized theory of water waves is used to examine the diffraction of an incident wave by a permeable breakwater that consists of a number of distinct elements. Under the assumption that the wavelength is much greater than the thickness, each element is replaced by a thin structure and the permeability is modeled by a suitable boundary condition applied on its surface. The diffracted wave field is obtained by the solution of an integral equation and results are presented to illustrate the effects of permeability and of the characteristics of the incident wave.
\end{abstract}

\section{Introduction}

Many beaches throughout the world are protected by a breakwater that is made up of a line of separated elements that are parallel to the shore (see King et al. 2000, Thomalla and Vincent 2003, and the references therein). The present work provides a simplified method for the calculation of the wave field around such a "segmented breakwater" that is made up of a number of distinct elements. The elements are assumed to be permeable, to stand in water of constant depth, and the wavelength of the incident waves is taken to be much greater than the thickness of the breakwaters so that each may be modelled by a thin vertical structure. With these assumptions the depth dependence can be factored out and the problem reduced to one of two-dimensional wave diffraction by line segments.

The two-dimensional problem of wave diffraction by thin impermeable structures has received considerable attention and references to this body of work can be found in the text by Linton and

*Professor of Applied Mathematics, Department of Mathematical Sciences, Loughborough University, Loughborough, Leicestershire LE11 3TU, U.K. 
McIver (2001). Of particular relevance to the present work is the diffraction of waves by a detached, impermeable, thin breakwater which has been treated, for example, by Stiassnie and Dagan (1972) using an expansion in terms of Mathieu functions, and by Gilbert and Brampton (1985) who formulated the problem in terms of an integral equation. More recently, diffraction of a wave group by such a detached breakwater has been investigated by Filianoti (2000). Mathematically related problems in the theory of electromagnetic waves have been studied extensively and sophisticated methods of solution developed; a recent reference to this body of work is Tsalamengas (1998).

Permeable structures can be treated by adopting a boundary condition based on the formulation of Sollitt and Cross (1972) for time-harmonic motion in a porous medium. This boundary condition has been used to solve the problems of wave diffraction by a semi-infinite breakwater (Yu 1995, McIver 1999) and by a gap in an infinite breakwater (Bowen and McIver 2002). Stiassnie and Dagan (1972) investigated a detached, permeable breakwater, but restricted their attention to a special case in which the permeability varies along the breakwater in a particular way. In the present work the boundary condition is used in a treatment of a segmented breakwater. An integral equation is obtained by an application of Green's theorem and a method described for its numerical solution. In addition a simplified procedure, based on the assumption that the breakwater segments are widely separated, is obtained and this allows an approximation to the solution for any number of segments to be determined once the solution for a single segment is known. Results are presented to illustrate the effects of variations in the incident wave characteristics on breakwaters with one or more segments.

\section{Formulation}

An arrangement of $N$ breakwater segments stands in water of constant depth $h$; the horizontal cross sections and the physical properties of each segment are uniform throughout the water depth. The length of the incident wave is assumed to be much greater than the breakwater thickness, and hence the thickness may be neglected and each segment modelled as a thin permeable structure. The model of such a structure used here is based on the work of Sollitt and Cross (1972) and is described in detail by $\mathrm{Yu}(1995)$.

The fluid motion is assumed to be time harmonic with angular frequency $\omega$ and to be of sufficiently small amplitude for linear theory to be applicable. Exterior to the breakwater the water is assumed to be inviscid and incompressible and the motion to be irrotational and hence it 


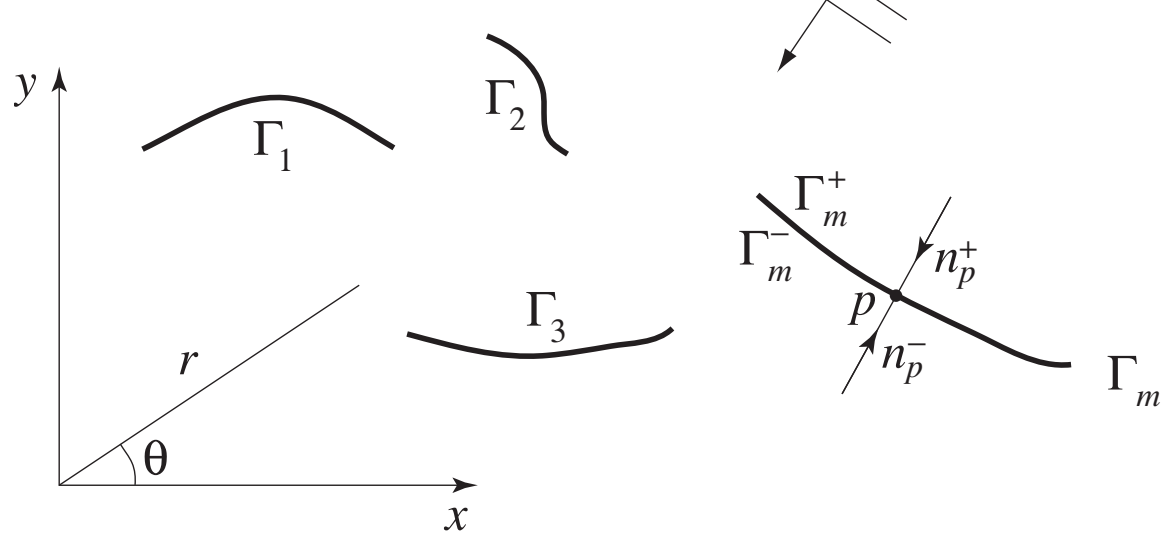

Figure 1: Coordinate systems.

may be described by a velocity potential.

Cartesian coordinates $x, y$ and $z$ are chosen with origin in the mean free surface so that the $x$ and $y$ axes lie in a horizontal plane and the $z$ axis is directed vertically upwards. An incident wave with amplitude $A$ and wave number $k$ propagates at an angle $\alpha$ to the $x$ axis (see figure 1 ), where $k$ is related to the frequency $\omega$ through the dispersion relation

$$
\omega^{2}=g k \tanh k h
$$

and $g$ is the acceleration due to gravity. Because of the assumptions on the uniformity of the breakwater in the vertical direction, the $z$ dependence may be removed from the problem by writing the velocity potential for the flow as

$$
\Phi(x, y, z, t)=\operatorname{Re}\left\{\frac{-\mathrm{i} g A}{\omega \cosh k h} \phi_{\mathrm{T}}(x, y) \cosh k(z+h) \mathrm{e}^{-\mathrm{i} \omega t}\right\}
$$

where the complex-valued function $\phi_{\mathrm{T}}(x, y)$ satisfies the Helmholtz equation

$$
\frac{\partial^{2} \phi_{\mathrm{T}}}{\partial x^{2}}+\frac{\partial^{2} \phi_{\mathrm{T}}}{\partial y^{2}}+k^{2} \phi_{\mathrm{T}}=0
$$

in the fluid domain exterior to the breakwater.

The problem has therefore been reduced to one in the $x, y$ plane and a plan view of the geometry is shown in figure 1, where the cross section of segment $m$ is denoted by $\Gamma_{m}$. A typical segment $\Gamma_{m}$ has two sides, denoted by $\Gamma_{m}^{+}$and $\Gamma_{m}^{-}$, and at a point $p$ on $\Gamma_{m}$ there are two normals directed out of the fluid into the segment and these are denoted by $n_{p}^{+}$and $n_{p}^{-}$. Throughout this work the lower 
case letters $p$ and $q$ are used to denote points on the surface of the breakwater, and the upper case letters $P$ and $Q$ to denote points within the fluid. In addition to the Cartesian coordinates defined above, in the horizontal plane standard polar coordinates $(r, \theta)$ will also be used (see figure 1).

On each breakwater segment conservation of mass requires

$$
\frac{\partial \phi_{\mathrm{T}}}{\partial n_{p}^{+}}\left(p^{+}\right)=-\frac{\partial \phi_{\mathrm{T}}}{\partial n_{p}^{-}}\left(p^{-}\right), \quad p \in \Gamma_{m}, m=1,2, \ldots, N .
$$

Here the superscript "+" ("-") on $p$ indicates a limit is taken as $\Gamma_{m}^{+}\left(\Gamma_{m}^{-}\right)$is approached from the fluid. In addition, the permeability conditions

$$
\frac{\partial \phi_{\mathrm{T}}}{\partial n_{p}^{+}}\left(p^{+}\right)=-\beta\left[\phi_{\mathrm{T}}(p)\right], \quad p \in \Gamma_{m}, m=1,2, \ldots, N,
$$

are to be satisfied, where

$$
[F(p)]=F\left(p^{+}\right)-F\left(p^{-}\right)
$$

is the jump in a quantity $F$ across the breakwater at the point $p$. The conditions (5) state that, at all points on a segment, the velocity of the flow through the structure is proportional to the pressure difference across it. The complex parameter

$$
\beta=\frac{-\mathrm{i} \epsilon}{b(f-\mathrm{i} s)}
$$

where $\epsilon$ is the porosity of the breakwater, $f$ is a non-dimensional friction coefficient, and $s$ is a nondimensional inertia coefficient. Here $\beta$ is assumed to be a constant, but it would be straightforward to modify the present formulation to allow $\beta$ to vary along each breakwater in a prescribed way.

The total potential $\phi_{\mathrm{T}}$ is written as

$$
\phi_{T}(x, y)=\phi_{\mathrm{I}}(x, y)+\phi(x, y)
$$

where the incident wave potential

$$
\phi_{\mathrm{I}}(x, y)=\mathrm{e}^{-\mathrm{i} k r \cos (\theta-\alpha)}
$$

and the potential $\phi$ for the diffracted wave field satisfies the radiation condition

$$
\lim _{r \rightarrow \infty} r^{\frac{1}{2}}\left(\frac{\partial \phi}{\partial r}-\mathrm{i} k \phi\right)=0
$$

The aim is to determine $\phi$ throughout the fluid domain and this is done through the solution of the integral equation obtained in the next section. 


\section{Derivation of an integral equation}

The derivation and numerical solution of the integral equation given here is based on results for a similar integral equation described in section 4.3 of Linton and McIver (2001). A suitable integral equation for the solution of the present problem is obtained through an application of Green's theorem to the potential $\phi$ for the diffracted field and the Green's function

$$
\phi_{0}(P, Q) \equiv \frac{1}{4 \mathrm{i}} H_{0}^{(1)}\left(k R_{P Q}\right) .
$$

Here $H_{n}^{(1)}$ denotes the Hankel function of the first kind and order $n, P$ and $Q$ are respectively the field and source points, and $R_{P Q}$ is the distance of $P$ from $Q$. For a field point $P$ with coordinates $(x, y)$ and a source point $Q$ with coordinates $(\xi, \eta)$ then $R_{P Q}^{2}=(x-\xi)^{2}+(y-\eta)^{2}$. Note that $\phi_{0}$ is a solution of the Helmholtz equation (3) at all points in the fluid domain $P \neq Q$. The application of Green's theorem over the fluid region exterior to the breakwater yields the representation

$$
\phi(P)=\int_{\Gamma}[\phi(q)] \frac{\partial \phi_{0}}{\partial n_{q}^{+}}(P, q) \mathrm{d} s_{q},
$$

where $\Gamma$ is the union of all breakwater segments, $s_{q}$ denotes arc length as $q$ is varied along $\Gamma$, and the integrand has been simplified using the continuity condition (4) and continuity of the derivatives of $\phi_{0}$. It remains to satisfy the condition (5) and this yields

$$
\frac{\partial \phi_{I}}{\partial n_{p}^{+}}+\beta[\phi(p)]=-\frac{\partial \phi}{\partial n_{p}^{+}}=-\frac{\partial}{\partial n_{p}^{+}} \int_{\Gamma}[\phi(q)] \frac{\partial \phi_{0}}{\partial n_{q}^{+}}(p, q) \mathrm{d} s_{q}, \quad p \in \Gamma,
$$

or

$$
\beta[\phi(p)]+\mathcal{f}_{\Gamma}[\phi(q)] \frac{\partial^{2} \phi_{0}}{\partial n_{p}^{+} \partial n_{q}^{+}}(p, q) \mathrm{d} s_{q}=-\frac{\partial \phi_{I}}{\partial n_{p}^{+}}, \quad p \in \Gamma,
$$

where the normal derivative has been taken under the integration sign by interpreting the integral as a finite-part integral. This interpretation is required because of the appearance of a strong singularity in the integrand that arises from the second derivative of the Green's function $\phi_{0}$. For a sufficiently smooth function $F$ a finite-part integral is defined by

$$
f_{a}^{b} \frac{F(t)}{(s-t)^{2}} \mathrm{~d} t=\lim _{\epsilon \rightarrow 0}\left\{\int_{a}^{s-\epsilon} \frac{F(t)}{(s-t)^{2}} \mathrm{~d} t+\int_{s+\epsilon}^{b} \frac{F(t)}{(s-t)^{2}} \mathrm{~d} t-\frac{2 F(s)}{\epsilon}\right\} .
$$

Equation (13) is an integral equation for the unknown jump in potential $[\phi(p)]$ and once this is known the potential everywhere in the fluid region follows from (11). Technical details related to the appearance of a finite-part integral in equation (13) may be found in Martin and Rizzo (1989); however a detailed knowledge of finite-part integrals is not needed to obtain numerical solutions of equation (13) in the way that will be described below. 


\section{Segments in a line}

In this section the general formulation given above is specialized to the case of breakwater made up of $N$ identical straight segments of length $2 a$ all positioned on the $x$ axis. Segment $\Gamma_{m}$ has its center at $(x, y)=\left(x_{m}, 0\right)$ and is parameterized so that $\Gamma_{m}=\left\{\left(x_{m}+a u, 0\right), u \in[-1,1]\right\}$. With this configuration the normal coordinates $n_{p}^{+}$and $n_{q}^{+}$are respectively equivalent to $y$ and $\eta$ and

$$
\frac{\partial^{2} \phi_{0}}{\partial n_{p}^{+} \partial n_{q}^{+}}(p, q)=\frac{k}{4 \mathrm{i} R_{p q}} H_{1}^{(1)}\left(k R_{p q}\right)
$$

(this makes explicit the highly singular nature of the integrand in equation (13)). If for a point $p$ on segment $m$ the jump in potential $[\phi(p)]$ is written as $\mathcal{P}_{m}(u), u \in[-1,1]$, then the integral equation (13) becomes

$$
\begin{aligned}
& \frac{\mathrm{i} \beta}{k} \mathcal{P}_{m}(u)+\frac{1}{4} \sum_{n=1}^{N} \mathcal{f}_{-1}^{1} \mathcal{P}_{n}(v) \frac{H_{1}^{(1)}\left(k a\left|\left(x_{m}-x_{n}\right) / a+u-v\right|\right.}{\left|\left(x_{m}-x_{n}\right) / a+u-v\right|} \mathrm{d} v \\
& \quad=\sin \alpha \mathrm{e}^{-\mathrm{i} k a\left(x_{m} / a+u\right) \cos \alpha}, \quad m=1,2, \ldots, N, \quad u \in[-1,1] .
\end{aligned}
$$

For a breakwater lying on the $x$ axis it may be shown (see section 4.7.2 of Linton and McIver 2001) that, for any point in the fluid, $\phi(x,-y)=-\phi(x, y)$ and therefore $\mathcal{P}_{m}(u)=2 \phi\left(p^{+}\right)$for each $p^{+}$on $\Gamma_{m}$. It also follows that for any point $(x, 0)$ not on any breakwater $\phi(x, 0)=0$ and, for all $m, \mathcal{P}_{m}(u) \rightarrow 0$ as $u \rightarrow \pm 1$. The derivatives of $\phi$ have square root singularities at the ends of the breakwaters and $\mathcal{P}_{m}(u)$ has zeros of order one half at $u= \pm 1$. The Chebyshev polynomials of the second kind $\left\{U_{s}(u) ; s=0,1,2, \ldots\right\}$ are complete over $(-1,1)$ with weight function $\left(1-u^{2}\right)^{1 / 2}$ and it is therefore appropriate to seek approximations in the form

$$
\mathcal{P}_{m}(u)=\left(1-u^{2}\right)^{1 / 2} \sum_{s=0}^{S} a_{m s} U_{s}(u), \quad m=1,2, \ldots N,
$$

where $S$ is an integer and the coefficients $a_{m s}$ are to be found. Substitution of (17) into the integral equation (16) gives

$$
\begin{aligned}
& \frac{\mathrm{i} \beta}{k}\left(1-u^{2}\right)^{1 / 2} \sum_{s=0}^{S} a_{m s} U_{s}(u)+\frac{1}{4} \sum_{s=0}^{S} a_{m s} f_{-1}^{1}\left(1-v^{2}\right)^{1 / 2} U_{s}(v) \frac{H_{1}^{(1)}(k a|u-v|}{|u-v|} \mathrm{d} v \\
& +\frac{1}{4} \sum_{n=1, n \neq m}^{N} \sum_{s=0}^{S} a_{n s} \int_{-1}^{1}\left(1-v^{2}\right)^{1 / 2} U_{s}(v) \frac{H_{1}^{(1)}\left(k a\left|\left(x_{m}-x_{n}\right) / a+u-v\right|\right.}{\left|\left(x_{m}-x_{n}\right) / a+u-v\right|} \mathrm{d} v \\
& =\sin \alpha \mathrm{e}^{-\mathrm{i} k a\left(x_{m} / a+u\right) \cos \alpha}, \quad m=1,2, \ldots, N, \quad u \in[-1,1] .
\end{aligned}
$$


The last summation in (18) involves integrals with non-singular integrands and the finite-part integral reverts to a standard integral. To obtain a set of simultaneous equations for the coefficients $a_{m s}$ from an equation of this type, Parsons and Martin (1992) suggest collocation at the points

$$
u_{s}=\cos \left[\frac{(2 s+1) \pi}{2 S+2}\right], \quad s=0,1, \ldots S
$$

which are the zeros of the Chebyshev polynomials of the first kind.

The evaluation of the finite-part integrals in (18) may be carried out with the aid of the result

$$
f_{-1}^{1} \frac{\left(1-v^{2}\right)^{1 / 2} U_{s}(v)}{(u-v)^{2}} \mathrm{~d} v=-\pi(s+1) U_{s}(v)
$$

(Frenkel 1983, equation 33) and the introduction of

$$
L(z)=H_{1}^{(1)}(z)+\frac{2 \mathrm{i}}{\pi z}-\frac{\mathrm{i}}{\pi} z \log z
$$

which has the property that $L(z) / z$ is non-singular as $z \rightarrow 0$. It follows that

$$
\begin{aligned}
f_{-1}^{1}\left(1-v^{2}\right)^{1 / 2} U_{s}(v) & \frac{H_{1}^{(1)}(k a|u-v|}{|u-v|} \mathrm{d} v=\frac{2 \mathrm{i}}{k a}(s+1) U_{s}(u) \\
& +\frac{\mathrm{i} k a}{\pi} f_{-1}^{1}\left(1-v^{2}\right)^{1 / 2} U_{s}(v) \log (k a|u-v|) \mathrm{d} v \\
& +\int_{-1}^{1}\left(1-v^{2}\right)^{1 / 2} U_{s}(v) \frac{L(k a|u-v|)}{|u-v|} \mathrm{d} v
\end{aligned}
$$

where the bar on the integral sign indicates a principal-value integral. This last integral can be evaluated using a standard adaptive integration routine after first subtracting out the singularity from the integrand (Antia 2002, section 6.6.5). The integral involving $L$ and the remaining integrals in (18) can all be evaluated accurately and efficiently using Gauss-Chebyshev quadrature based on Chebyshev polynomials of the second kind (Antia 2002, section 6.5.2).

It is worth noting that the angle of wave incidence $\alpha$ appears only on the right-hand side of equation (18). Thus, for a given breakwater configuration, the expansion coefficients can be determined for all $\alpha$ from a single "inversion" of the matrix appearing in the equivalent set of simultaneous equations. For the computations reported below the LU decomposition of the matrix was computed and solutions found for each $\alpha$ by back substitution (Antia 2002, section 3.3).

\section{Calculation of the wave field}

Once the jump in potential across each breakwater has been determined through the solution of the integral equation the potential everywhere in the fluid domain follows from (11) which, with 
the aid of (17) and the result

$$
\frac{\partial \phi_{0}}{\partial n_{q}^{+}}(P, q)=\frac{1}{4} \mathrm{i} k y \frac{H_{1}^{(1)}\left(k R_{P q}\right)}{R_{P q}}
$$

may be written

$$
\begin{aligned}
\phi(x, y) & =\frac{1}{4} \mathrm{i} k a y \sum_{m=1}^{N} \int_{-1}^{1} \mathcal{P}_{m}(v) \frac{H_{1}^{(1)}\left(k R_{m}\right)}{R_{m}} \mathrm{~d} v \\
& =\frac{1}{4} \mathrm{i} k a y \sum_{m=1}^{N} \sum_{s=0}^{S} a_{m s} \int_{-1}^{1}\left(1-v^{2}\right)^{1 / 2} U_{s}(v) \frac{H_{1}^{(1)}\left(k R_{m}\right)}{R_{m}} \mathrm{~d} v, \quad y \neq 0,
\end{aligned}
$$

where $R_{m}^{2}=\left(x-x_{m}-a v\right)^{2}+y^{2}$. The integrals appearing in equation (24) may be evaluated numerically using Gauss-Chebyshev quadrature. For all $m$, on the surface $\Gamma_{m}^{ \pm}$of segment $m$ the potential is given by $\pm P_{m}\left(\left(x-x_{m}\right) / a\right) / 2$ and elsewhere on $y=0$ it is zero.

In the far field the expression for $\phi$ can be simplified using the result

$$
H_{1}^{(1)}(z) \sim\left(\frac{2}{\pi z}\right)^{1 / 2} \mathrm{e}^{\mathrm{i}(z-3 \pi / 4)} \quad \text { as } \quad z \rightarrow \infty
$$

(Abramowitz and Stegun, 1964). Now as $r=\left(x^{2}+y^{2}\right)^{1 / 2} \rightarrow \infty$

$$
\begin{aligned}
R_{m}=\left[\left(x-x_{m}-a v\right)^{2}+y^{2}\right]^{1 / 2} & =r\left(1-2 x\left(x_{m}+a v\right) / r+O\left(r^{-2}\right)\right)^{1 / 2} \\
& =r-\left(x_{m}+a v\right) \cos \theta+O\left(r^{-1}\right)
\end{aligned}
$$

so that, retaining only the first approximation,

$$
\phi(x, y) \sim \frac{\mathrm{e}^{\mathrm{i}(k r-3 \pi / 4)}}{(2 \pi k r)^{1 / 2}} G(\theta, \alpha) \quad \text { as } \quad k r \rightarrow \infty
$$

where the diffraction coefficient

$$
G(\theta, \alpha)=\frac{1}{2} \mathrm{i} k a \sin \theta \sum_{s=0}^{S}\left\{\int_{-1}^{1}\left(1-v^{2}\right)^{1 / 2} U_{s}(v) \mathrm{e}^{-\mathrm{i} k a v \cos \theta} \mathrm{d} v \sum_{m=1}^{N} a_{m s} \mathrm{e}^{-\mathrm{i} k x_{m} \cos \theta}\right\}
$$

and again the integral in (28) is readily evaluated using Gauss-Chebyshev quadrature. It may be shown using a similar argument to that given in Bowen and McIver (2002) that the reciprocity relation

$$
G(\theta, \alpha)=G(\alpha, \theta)
$$

is satisfied. 


\section{Wide-spacing approximation}

A simplified solution method can be obtained if the breakwater segments are assumed to be sufficiently widely spaced for terms in (18) that involve distances between points on different segments to be neglected. This leads to the modified system

$$
\begin{aligned}
\frac{\mathrm{i} \beta}{k}\left(1-u^{2}\right)^{1 / 2} \sum_{s=0}^{S} a_{s} U_{s}(u)+\frac{1}{4} \sum_{s=0}^{S} a_{s} \oint_{-1}^{1}\left(1-v^{2}\right)^{1 / 2} U_{s}(v) & \frac{H_{1}^{(1)}(k a|u-v|}{|u-v|} \mathrm{d} v \\
& =\sin \alpha \mathrm{e}^{-\mathrm{i} k a u \cos \alpha}, \quad u \in[-1,1] .
\end{aligned}
$$

where the coefficients $a_{s}$ are those for a single segment centered on $x=0$. The corresponding coefficients for the full problem are recovered from

$$
a_{m s}=a_{s} \mathrm{e}^{-\mathrm{i} k x_{m} \cos \alpha}
$$

Under the wide-spacing approximation there is no significant simplification of (24). However, in the expression for the diffraction coefficient (28) the series decouple to give

$$
G(\theta, \alpha)=\frac{1}{2} \mathrm{i} k a \sin \theta\left\{\sum_{s=0}^{S} a_{s} \int_{-1}^{1}\left(1-v^{2}\right)^{1 / 2} U_{s}(v) \mathrm{e}^{-\mathrm{i} k a v \cos \theta} \mathrm{d} v\right\}\left\{\sum_{m=1}^{N} \mathrm{e}^{-\mathrm{i} k x_{m}(\cos \theta+\cos \alpha)}\right\}
$$

\section{Results}

Results for the diffraction of waves by a single solid breakwater are available elsewhere (see, for example, Stiassnie and Dagan 1972, Gilbert and Brampton 1985, or section 4.7 of Linton and McIver 2001 and the references therein). The effects of permeability on the diffraction coefficient $G$, defined in equation (28), is illustrated in figure 2 where the modulus is given as a function of observation angle $\theta$ for a variety of wavenumbers $k a$ and angles of wave incidence $\alpha$. The particular non-zero value of the permeability parameter $\beta$ chosen in these computations is that used by $\mathrm{Yu}$ (1995), McIver (1999), and Bowen and McIver (2002). It can be seen that permeability acts to reduce the amplitude of diffraction effects although the main features of the diffraction pattern are preserved.

The wide-spacing approximation described above computes the interactions between breakwater segments by combining results for diffraction by a single segment, with allowance made for the phase differences arising from the positions of different segments. No account is taken of the effects on neighboring segments of the waves diffracted by each segment. For a single breakwater 

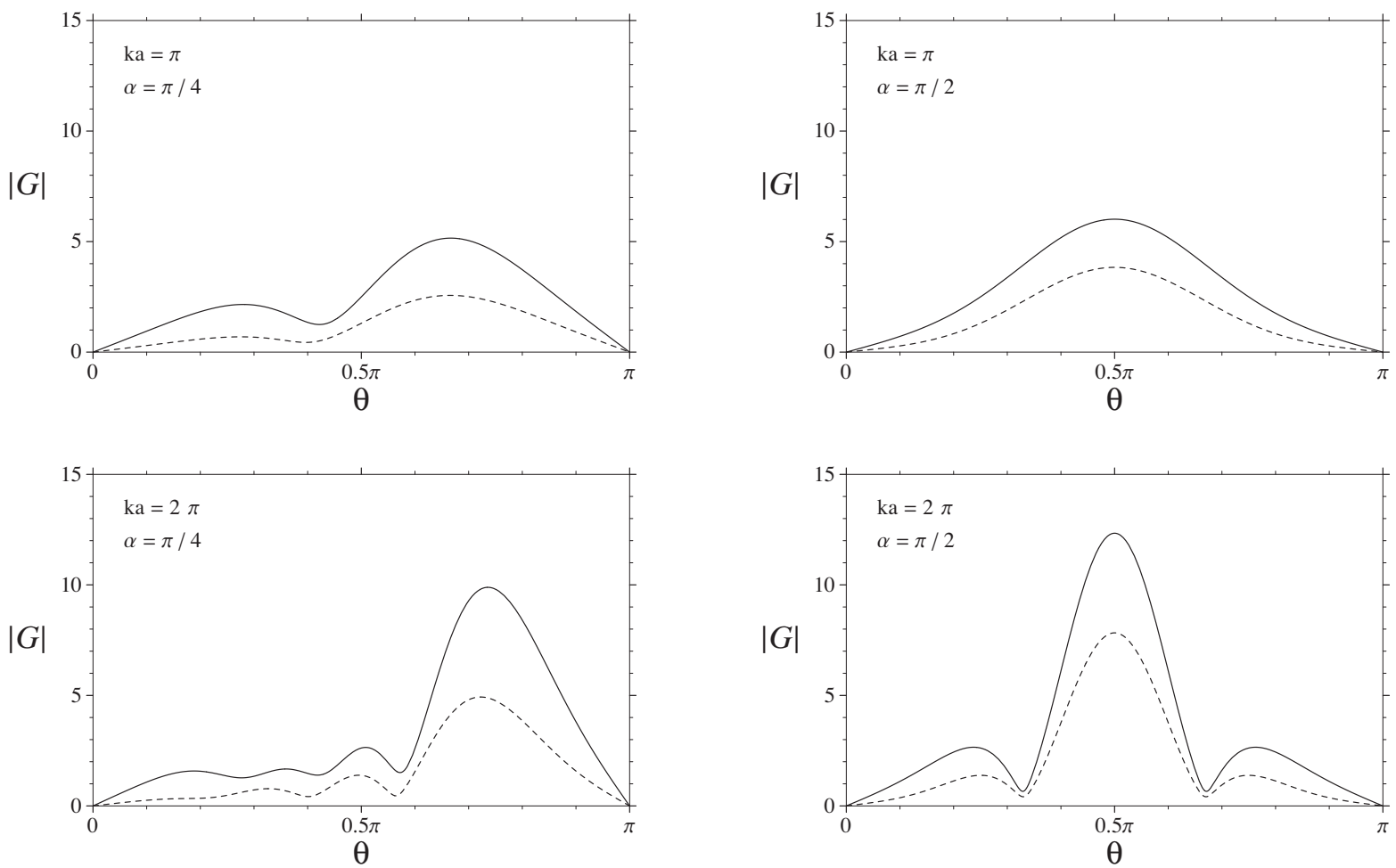

Figure 2: Modulus of diffraction coefficient $|G(\theta, \alpha)|$ v. observation angle $\theta$ for various incident

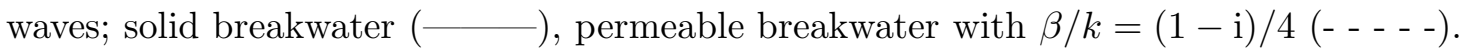



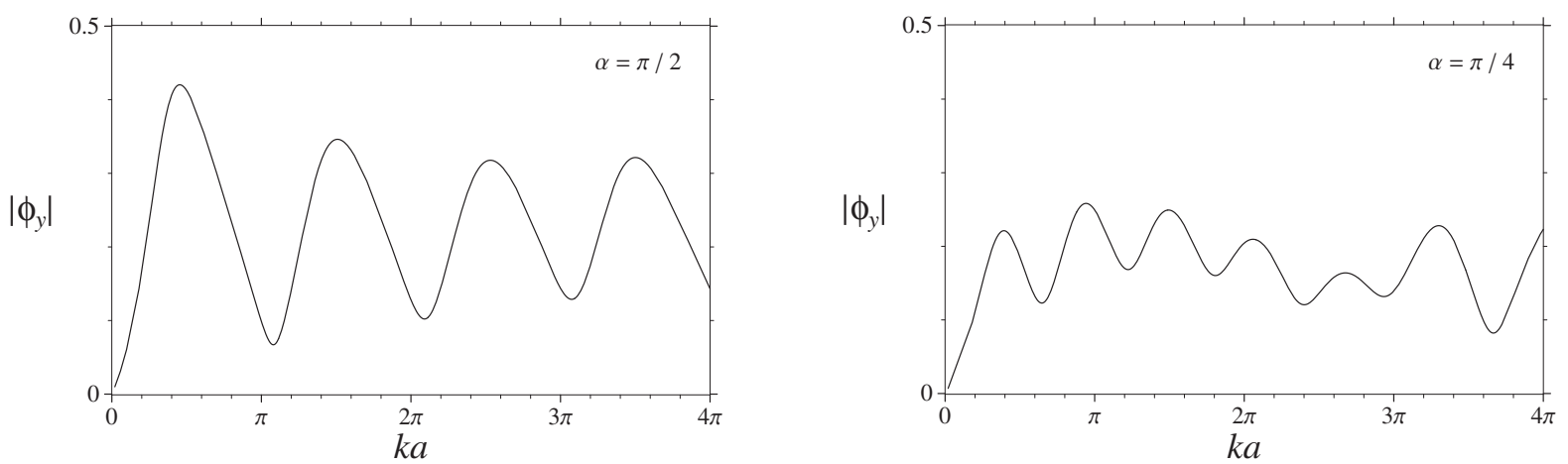

Figure 3: Single solid breakwater: modulus of velocity $\left|\phi_{y}\right|$ at $(x, y)=(2 a, 0)$ v. $k a$ for two angles of wave incidence $\alpha$.

the diffracted field $\phi$ is zero on the line of the breakwater (see the discussion following equation 16) but the fluid velocity $\phi_{y}$ perpendicular to this line is non zero and it is this that generates interactions between segments of a multi-element breakwater. The magnitude of the velocity $\left|\phi_{y}\right|$ is singular at the breakwater tip and, into the fluid, decays monotonically with distance from the tip. However, the rate of decay varies with both the wavenumber and angle of the incident waves and this effect is illustrated in figure 3 for a solid breakwater $(\beta=0)$ by plotting $\left|\phi_{y}\right|$ at the point $(x, y)=(2 a, 0)$ as a function of wave number for two angles of wave incidence. For $\alpha=\pi / 2$ the strongest interactions between segments of a multi-element breakwater can be expected for $k a \approx \pi / 2$ (so that the wavelength is about twice the length of a segment), and weak interactions can be expected for $k a$ close to an integer multiple of $\pi$. For $\alpha=\pi / 4$ there are smaller variations in $\left|\phi_{y}\right|$ and there will be a correspondingly reduced dependence on $k a$ of interactions between neighboring segments.

An indication of the accuracy that can be achieved by the wide-spacing approximation is given in figure 4 where the diffraction coefficient for a breakwater with two segments with centers at $x= \pm 1.1 a$ is presented. These results, for $\alpha=\pi / 2$, are consistent with the discussion of figure 3 and show how the strength of the interactions vary with $k a$. The accuracy of the wide-spacing approximation, of course, also depends on the spacing between segments and will improve with increasing spacing. For example, when the two segments have centers at $x= \pm 1.5 a$ (not shown here) the wide-spacing approximation to the diffraction coefficient is close to being graphically indistinguishable from the full calculation for those values of $k a$ and $\alpha$ used in figure 4 . From now on all results presented were calculated by the full method. It should be noted that for computations 

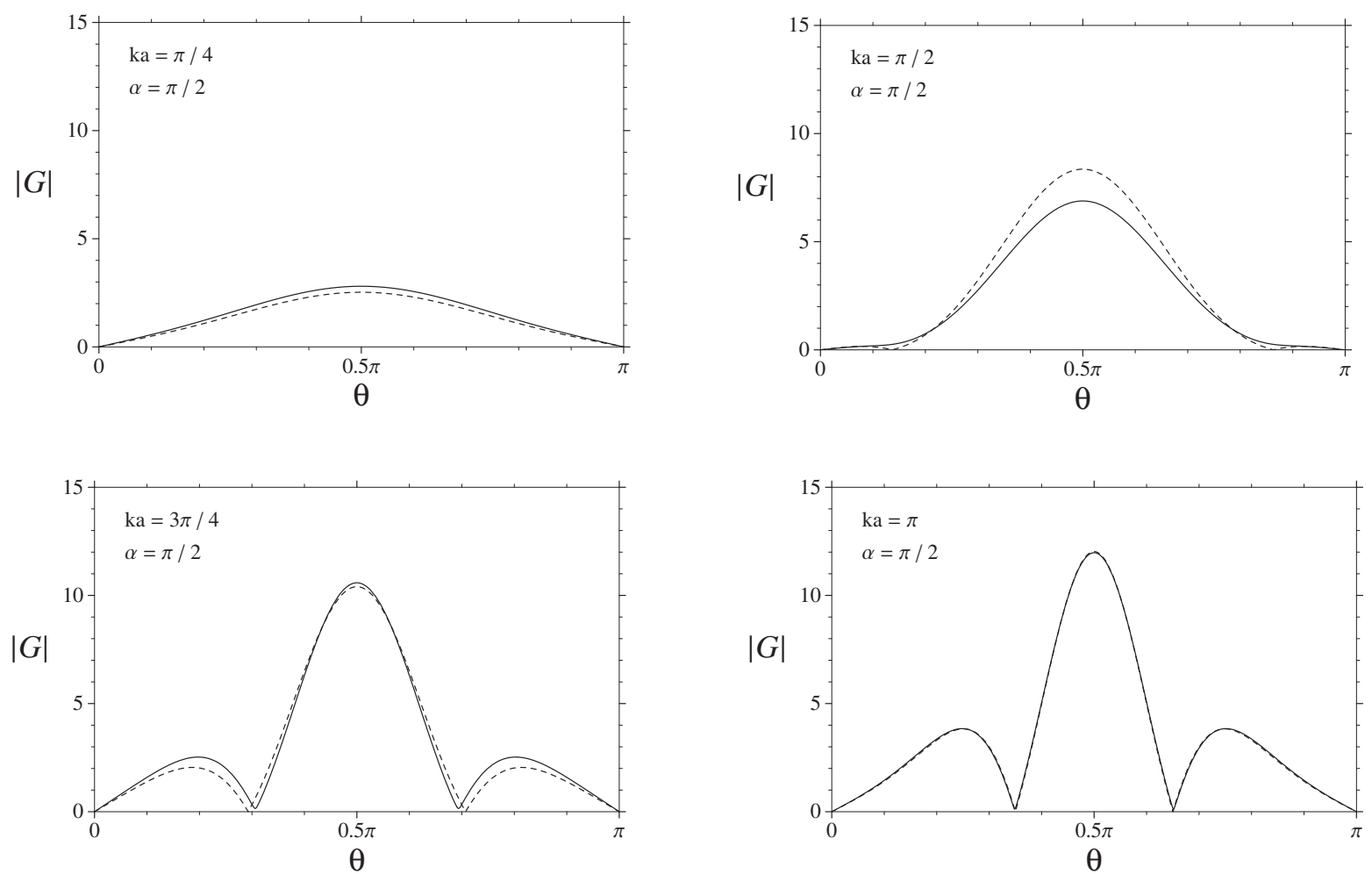

Figure 4: Two solid breakwaters with centers at $x= \pm 1.1 a$ : modulus of diffraction coefficient $|G(\theta, \alpha)|$ v. observation angle $\theta$ for incident waves with angle of incidence $\alpha=\pi / 2$ and various wavenumbers $k a$; exact solution ( - ), wide-spacing approximation (- - - - ). 

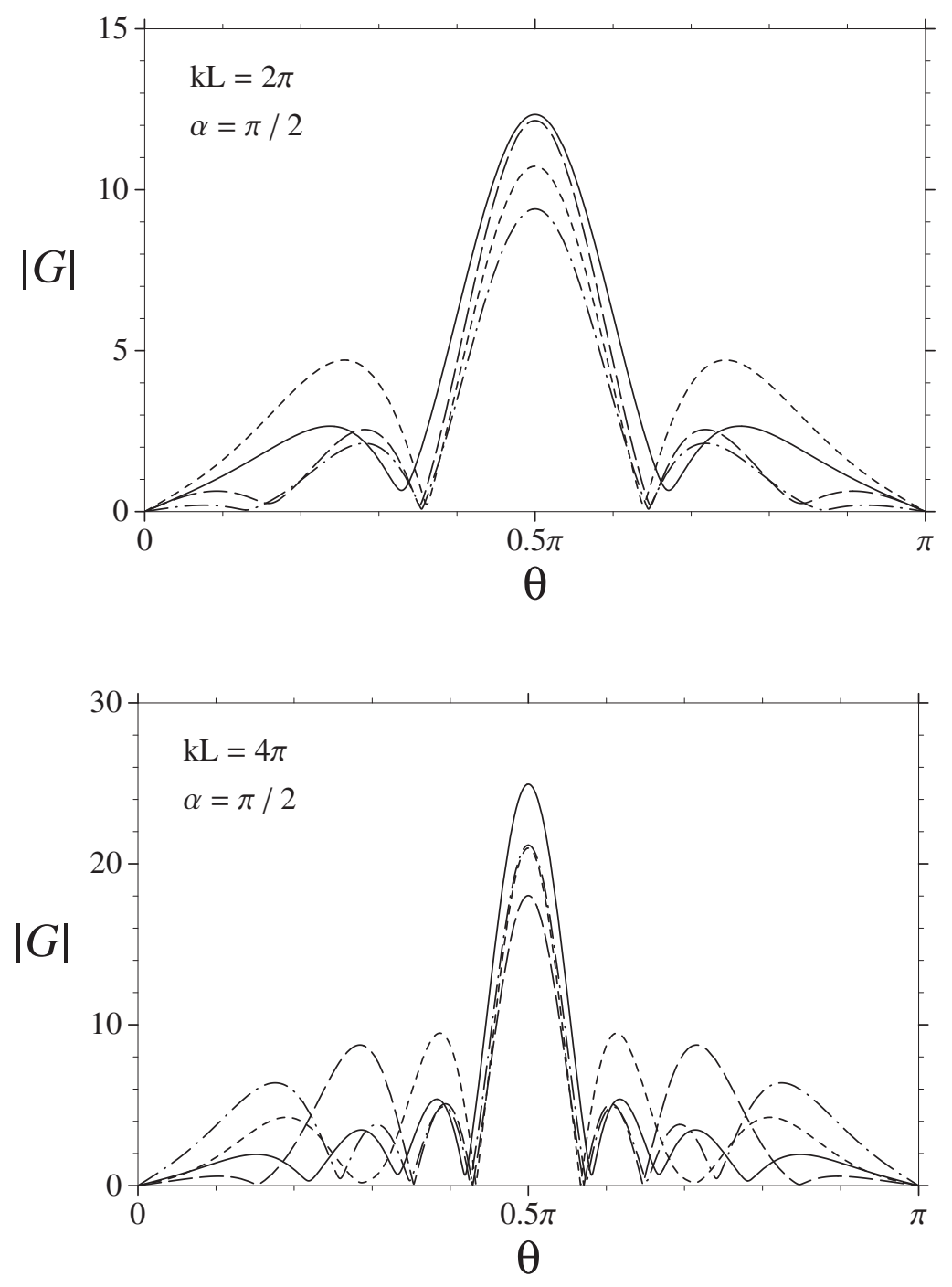

Figure 5: Solid breakwater of length $L$ with various numbers of segments: modulus of diffraction coefficient $|G(\theta, \alpha)|$ v. observation angle $\theta$ for incident waves with angle of incidence $\alpha=\pi / 2$ and two wavenumbers $k L$; one segment (- - ), two segments (- - - -), three segments ( $-\longrightarrow)$, four segments $(-\cdot-\cdot-)$.

of the distribution of $\phi$ within the vicinity of the breakwater there is little advantage in using the wide-spacing approximation as, at least for a modest number of breakwater segments, most of the computational effort is in computing $\phi$ rather than in solving for the expansion coefficients.

The effect on the diffraction coefficient of the number of segments in a breakwater of fixed length $2 L$ is shown in figure 5 . Each segment is of length $2 a$ and the gap between neighboring segments is $a$; thus for one, two, three and four segments the length parameter $L$ equals, respectively, $a, 5 a / 2$, 

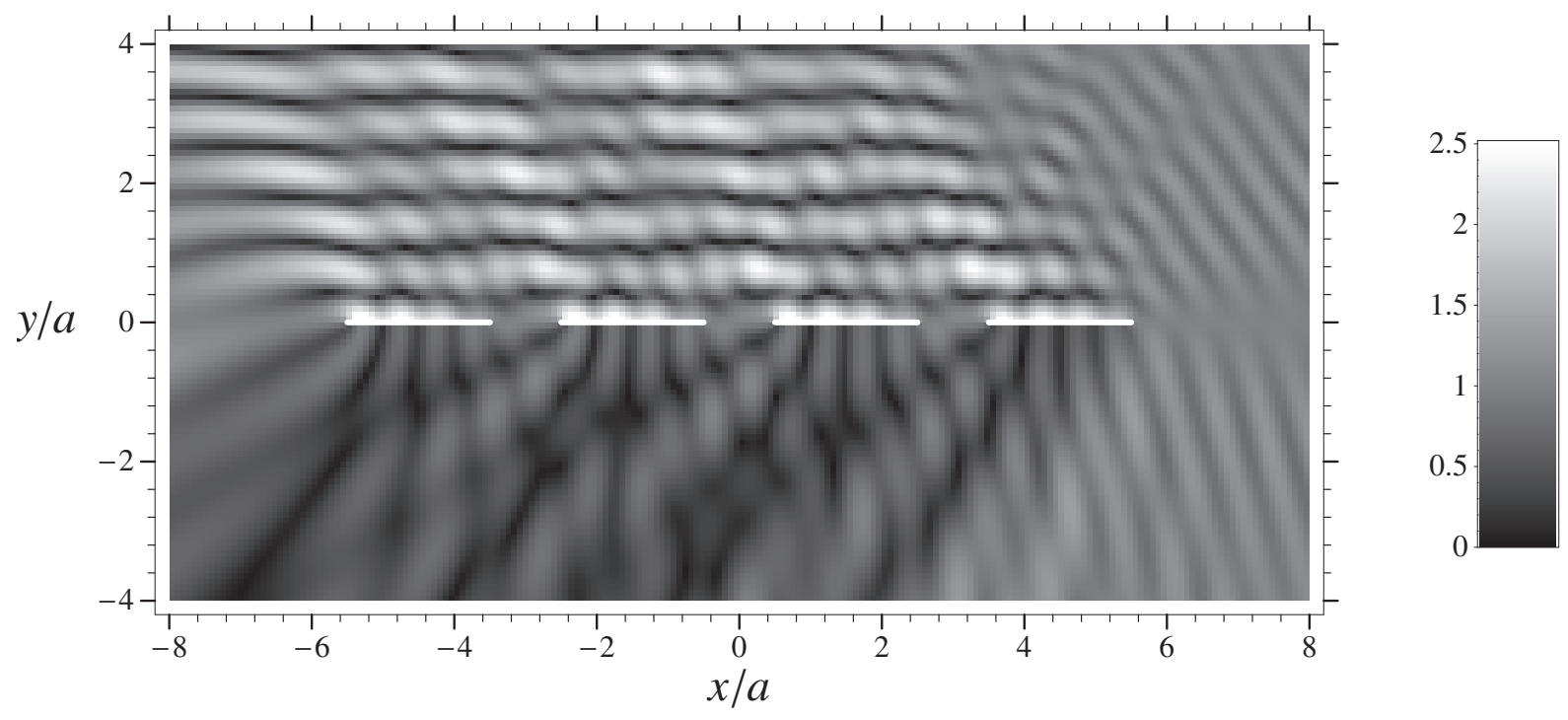

Figure 6: Density plot of $\left|\phi_{T}(x, y)\right|$; permeability $\beta / k=0$, wavenumber $k a=2 \pi$, angle of wave incidence $\alpha=\pi / 4$.

$4 a$, and $11 a / 2$. For normal wave incidence $\alpha=\pi / 2$, the main peak in the diffraction coefficient is reduced by the presence of gaps in the breakwater, although this is not monotonic with an increasing number of gaps. Perhaps the most significant differences in the diffraction pattern caused by the gaps are for angles of observation around $\pm \pi / 4$ from the normal to the breakwater. Clearly, the complexity of the wave pattern increases as the number of segments increases.

The final results presented here in figures 6 and 7 are for the distribution of $\left|\phi_{T}\right|$ (which is proportional to the amplitude of the free-surface oscillation at each point) in the vicinity of a breakwater. The geometrical configuration used in the computations is that of four segments each of length $2 a$ and separated by a distance $a$ (this is the four-segment configuration that is used in figure 5). In figure 6 the breakwater is impermeable and in figure 7 the breakwater is permeable. In both cases the incident wave has wavenumber $k a=2 \pi$ and angle of incidence $\alpha=\pi / 4$.

In the regions of figures 6 and 7 where the diffracted field is small, so that the solution is given approximately by the incident wave $\phi_{I}$, then $\left|\phi_{T}\right| \approx 1$ and there is almost uniform darkgrey shading. Striped regions of alternating high and low amplitude indicate standing waves; these appear most strongly on the side of the breakwater facing the incident waves. Overall the diffraction patterns observed in the two figures are very similar with the pattern produced by the impermeable barrier having a wider range of amplitudes. In both cases there are regions behind the breakwater where the amplitude of the fluid motion is comparable with that of the incident wave. 


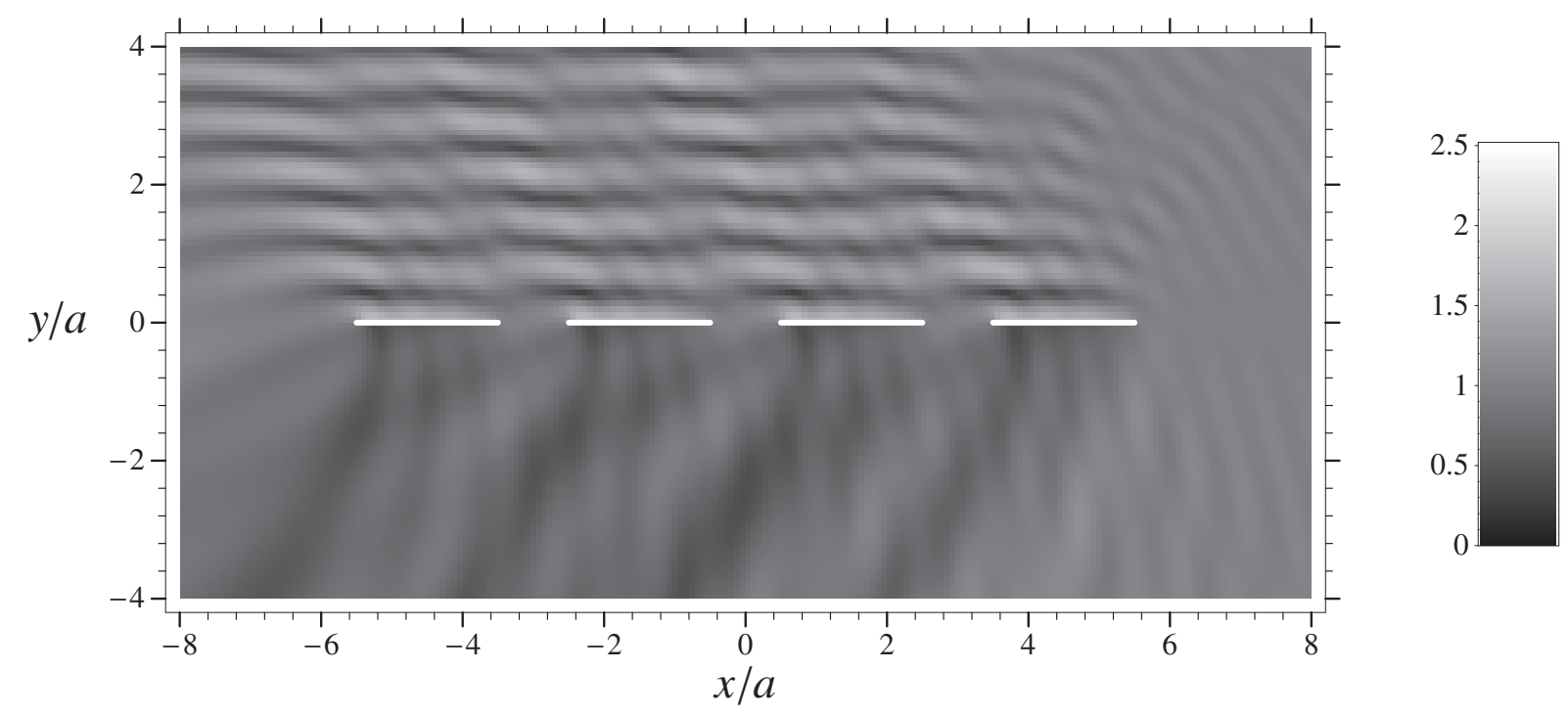

Figure 7: Density plot of $\left|\phi_{T}(x, y)\right|$; permeability $\beta / k=(1-\mathrm{i}) / 4$, wavenumber $k a=2 \pi$, angle of wave incidence $\alpha=\pi / 4$.

\section{References}

Abramowitz, M and Stegun, I. A. (1964) "Handbook of Mathematical Functions." National Bureau of Standards.

Antia, H. M. (2002) "Numerical Methods for Scientists and Engineers." Birkhäuser.

Bowen, M. K. and McIver, P. (2002) "Diffraction by a gap in an infinite permeable breakwater." J. Wtrwy., Port, Coast. and Ocean Engrg. ASCE, 125, 66-70.

Filianoti, P. (2000) "Diffraction of random wind-generated waves by a detached breakwater or breakwater gap." Ocean Engrg., 27, 1249-1263.

Frenkel, A. (1983) "External modes of two-dimensional thin scatterers." IEE Proc. H, Microwaves, Opt. E Antennas., 130, 209-214.

Gilbert, G. and Brampton, A. H. (1985) "The solution of two wave diffraction problems using integral equations." Technical Report IT 299, Hydraulics Research, Wallingford.

King, D. M., Cooper, N. J., Morfett, J. C. and Pope, D. J. (2000) "Application of offshore breakwaters to the UK: A case study at Elmer beach." J. Coast. Res., 16, 172-187. 
Linton, C. M. and McIver, P. (2001) "Handbook of Mathematical Techniques for Wave/Structure Interactions." Chapman \& Hall/CRC, Boca Raton.

Martin, P. A. and Rizzo, F. J. (1989) "On boundary integral equations for crack problems." Proc. Roy. Soc. Lond., A 421, 341-355.

McIver, P. (1999) "Water-wave diffraction by thin porous breakwater." J. Wtrwy., Port, Coast. and Ocean Engrg. ASCE, 125, 66-70.

Parsons, N. F. and Martin, P. A. (1992) "Scattering of water waves by submerged plates using hypersingular integral equations." Appl. Ocean Res., 14, 313-321.

Sollitt, C. K. and Cross, R. H. (1972) "Wave transmission through permeable breakwaters." Proc., 13th Conf. on Coast. Engrg., ASCE, 1827-1846.

Stiassnie, M. and Dagan, G. (1972) "Wave diffraction by detached breakwater." J. Wtrwys., Harbors and Coast. Engrg. Div. ASCE, 98, 209-224.

Thomalla, F. and Vincent, C. E. (2003) "Beach response to shore parallel breakwaters at Sea Palling, Norfolk, UK." Estuarine, Coast. Shelf Sci., 56, 203-212.

Tsalamengas, J. L. (1998) "Direct singular integral equation methods in scattering and propagation in strip- or slot-loaded structures." IEEE Trans. Antennas Propagat., 46, 1560-1570.

Yu, X. (1995) "Diffraction of water waves by porous breakwaters." J. Wtrwy., Port, Coast. and Ocean Engrg. ASCE, 121, 275-282. 